\title{
Palmitate- and C6 ceramide-induced Tnnt3 pre-mRNA alternative splicing occurs in a PP2A dependent manner
}

\author{
Adam J. Black ${ }^{1,2}$, Rudolf J. Schilder ${ }^{3}$ and Scot R. Kimball ${ }^{\text {** }}$
}

\begin{abstract}
Background: In a previous study, we showed that consumption of diets enriched in saturated fatty acids causes changes in alternative splicing of pre-mRNAs encoding a number of proteins in rat skeletal muscle, including the one encoding skeletal muscle Troponin T (Tnnt3). However, whether saturated fatty acids act directly on muscle cells to modulate alternative pre-mRNA splicing was not assessed. Moreover, the signaling pathway through which saturated fatty acids act to promote changes in alternative splicing is unknown. Therefore, the objective of the present study was to characterize the signaling pathway through which saturated fatty acids act to modulate Tnnt3 alternative splicing.

Methods: The effects of treatment of L6 myotubes with saturated (palmitate), mono- (oleate), or polyunsaturated (linoleate) fatty acids on alternative splicing of pre-mRNA was assessed using Tnnt3 as a marker gene.

Results: Palmitate treatment caused a two-fold change $(p<0.05)$ in L6 myotube Tnnt3 alternative splicing whereas treatment with either oleate or linoleate had minimal effects compared to control myotubes. Treatment with a downstream metabolite of palmitate, ceramide, had effects similar to palmitate on Tnnt3 alternative splicing and inhibition of de novo ceramide biosynthesis blocked the palmitate-induced alternative splicing changes. The effects of palmitate and ceramide on Tnnt3 alternative splicing were accompanied by a 40-50\% reduction in phosphorylation of Akt on S473. However, inhibition of de novo ceramide biosynthesis did not prevent palmitate-induced Akt dephosphorylation, suggesting that palmitate may act in an Akt-independent manner to modulate Tnnt3 alternative splicing. Instead, pre-treatment with okadaic acid at concentrations that selectively inhibit protein phosphatase 2A (PP2A) blocked both palmitate- and ceramide-induced changes in Tnnt3 alternative splicing, suggesting that palmitate and ceramide act through PP2A to modulate Tnnt3 alternative splicing.
\end{abstract}

Conclusions: Overall, the data show that fatty acid saturation level and ceramides are important factors modulating alternative pre-mRNA splicing through activation of PP2A.

Keywords: Alternative splicing, Fatty acids, Palmitate, Ceramides, Troponin T, Tnnt3, Protein phosphatase 2A

\section{Introduction}

Alternative splicing is a critical step in the processing of precursor mRNA (pre-mRNA) into mature mRNA that involves the selective removal of exons from a pre-mRNA. Alternative splicing can generate multiple mature mRNAs from a single gene thus allowing for a significant expansion of the transcriptome and a concomitant increase in proteome plasticity and functionality [1]. The regulation

\footnotetext{
* Correspondence: skimball@psu.edu

${ }^{1}$ Department of Cellular and Molecular Physiology, Penn State College of Medicine, H166, 500 University Drive, Hershey, PA 17033, USA Full list of author information is available at the end of the article
}

of alternative splicing is complex. In brief, it involves the dynamic interplay of cis- and trans-acting factors to coordinate the placement of the spliceosome, a large macromolecular complex responsible for the catalytic removal of introns and exons, onto a pre-mRNA [2-6]. Cis-acting factors are RNA sequence elements and thus are inherent to the pre-mRNA [7, 8], whereas trans-acting factors are splicing accessory proteins that bind cis-factors to facilitate splice site recognition $[6,9,10]$. In addition to being regulated through transcriptional mechanisms, trans-acting factors are also post-translationally modified by kinases and phosphatases $[11,12]$. Thus, the

(c) The Author(s). 2018 Open Access This article is distributed under the terms of the Creative Commons Attribution 4.0 International License (http://creativecommons.org/licenses/by/4.0/), which permits unrestricted use, distribution, and 
phosphorylation state of components of the splicing regulatory machinery is a critical determinant of whether or not an alternatively spliced exon is included in or excluded from a mature RNA.

One of the best characterized examples of phosphorylation-mediated regulation of alternative splicing is through the Akt signaling axis. Indeed, several trans-acting factors (i.e. serine-arginine-rich (SR) proteins and SR protein kinases) involved in pre-mRNA alternative splicing are substrates of Akt [4, 11-14]. The contribution of protein phosphatases (PP) to changes in trans-acting factor phosphorylation is less well studied. Protein phosphatases (PP) 1 and $2 \mathrm{~A}$ are required for the proper assembly and disassembly of spliceosomal components [15] and regulate alternative splicing by directly targeting trans-acting proteins or indirectly by modulating Akt phosphorylation $[16,17]$.

Troponin is a heterotrimeric complex that mediates calcium-induced sarcomere contraction [18]. The troponin $\mathrm{T}$ subunit links the calcium binding subunit, troponin $\mathrm{C}$, to tropomyosin, and therefore plays a critical role in calcium signaling to the sarcomere. We recently demonstrated that fast skeletal muscle troponin $\mathrm{T}$ (Tnnt3) pre-mRNA is alternatively spliced in rat gastrocnemius muscle in response to consumption of a high-fat diet prior to the onset of obesity. Interestingly, this effect was specific to diets enriched in lard, as diets enriched in mono- or polyunsaturated fatty acids had no effect on Tnnt3 alternative splicing [19]. A major difference between the three diets used in that study was the proportion of the saturated fat, palmitate, in the lard-enriched diet. Palmitate is a substrate commonly used in the biosynthesis of ceramides [20, 21], a family of sphingolipid second messengers that are induced in response to a variety of cellular stressors [22-24]. The above observations suggest that the diet-specific effects on Tnnt3 pre-mRNA alternative splicing are caused by specific types of fatty acids in the high-fat lard diet and/ or the intramuscular accumulation of ceramides. There is some precedent for this hypothesis, as previous work demonstrated that PP1 is necessary for the ceramideinduced changes in the alternative splicing of Bcl-xL and caspase-9 pre-mRNAs [24]. Other studies have reported diet- or obesity-induced changes in pre-mRNA alternative splicing [25-34], but little is known about the role of specific fatty acids in this process.

Though it is tempting to speculate that changes in skeletal muscle alternative splicing in the aforementioned studies were due to the accumulation of fatty acids or ceramides, hormonal feedback mechanisms controlling metabolic status complicate delineating mechanisms that modulate alternative splicing in vivo $[1,35]$. Hence, we employed an in vitro system to precisely control the exposure of muscle cells to fatty acids and ceramides to examine the effects on alternative splicing and potential signaling pathways involved in regulating this process. Therefore, using Tnnt3 as a marker gene in L6 myotubes we hypothesized that treatment with saturated fatty acids and ceramides would alter the pattern of alternative splicing while unsaturated fatty acids would have little to no effect.

\section{Methods}

\section{Fatty acid/BSA conjugation}

Palmitate, oleate, or linoleate (Sigma, St. Louis, MO) were dissolved in glass vials at $70{ }^{\circ} \mathrm{C}$ in a $50 \%$ Ethanol/ Dulbecco's phosphate buffered saline (DPBS) (Gibco, Thermo Fisher, Waltham, MA) solution and added to a solution of $10 \%$ fatty acid-free BSA (Sigma) that was pre-warmed to $55^{\circ} \mathrm{C}$. The fatty acid-BSA conjugates were mixed at $55^{\circ} \mathrm{C}$ for $20 \mathrm{~min}$ then filter sterilized through $0.45 \mu \mathrm{m}$ syringe filters (Millipore, Billerica, MA) and stored at $-20^{\circ} \mathrm{C}$ in glass vials.

\section{Cell culture}

L6 myoblasts $\left(1 \times 10^{5}\right.$; passage $\left.3-10\right)$ (ATCC, Manassas, VA) were seeded in six-well culture plates in DMEM (Gibco) containing 10\% FBS (Atlas Biologicals, Fort Collins, CO) and 1\% Pen/Strep (Gibco). When the L6 myoblasts reached $80-85 \%$ confluence (approximately $48 \mathrm{~h}$ post seeding) they were induced to differentiate to myotubes by replacing the media with DMEM containing $2 \%$ horse serum (Sigma) and 1\% Pen/Strep (differentiation media (DM)). DM was refreshed every $48 \mathrm{~h}$. At the end of the sixth day, fresh DM was added approximately $16 \mathrm{~h}$ prior to the addition of fatty acids, C6 ceramide (d18:1/6:0) (Avanti Polar Lipids, Alabaster, Alabama), or chemical inhibitors of de novo ceramide biosynthesis (myriocin) or PP2A (okadaic acid). For experiments using chemical inhibitors, L6 myotubes were pretreated for two hours with $50 \mathrm{nM}$ myriocin (Sigma), $15 \mathrm{nM}$ okadaic acid (LC Labs, Woburn, MA), or vehicle (methanol or DMSO, respectively) prior to the addition of $150 \mu \mathrm{M}$ fatty acid-BSA conjugate or $20 \mu \mathrm{M}$ C6 ceramide. Cells were treated with fatty acid-BSA conjugate or C6 ceramide for $24 \mathrm{~h}$ and collected for analysis eight days after the onset of differentiation.

\section{Western blot analysis}

L6 myotubes were washed once in cold phosphate buffered saline and harvested in lysis buffer as described previously [36]. Myotube samples were fractionated by SDS-PAGE using Bio-Rad precast Criterion gels (BioRad, Hercules, CA) as previously described [37]. Select PVDF membranes were stained with a reversible protein stain kit according to the manufacturer's instructions (Pierce, Thermo Fisher). Primary antibodies against 
phospho-Akt (S473) (\#4060; 1:2000), ERK1/2 (T202/ Y204) (\#9101; 1:1000), and GSK33 (S9) (\#5558; 1:1000), and total-Akt (\#9272; 1:1000), ERK1/2 (\#9102; 1:1000), and GSK3 $\beta$ (\#12456; 1:1000) were purchased from Cell Signaling (Danvers, MA). Anti-TNNT3 antibody (\#ILT12; $0.2 \mu \mathrm{g} / \mathrm{mL}$ ) was purchased from Developmental Studies Hybridoma Bank (Iowa City, IA). Western blot densitometry was quantitated using Image J Software (National Institutes of Health). Densitometry was normalized to a pooled sample of untreated myotubes collected in lysis buffer as above and run in duplicate on each gel.

\section{Tnnt3 splice form characterization and quantification}

L6 myotubes were collected in $1 \mathrm{~mL}$ TRIzol then stored at $-80^{\circ} \mathrm{C}$ until total RNA was extracted per manufacturer's instructions (Invitrogen, Thermo Fisher), with the exception that the precipitated RNA pellet underwent an additional $85 \%$ ethanol wash. One $\mu$ g of total RNA was reverse transcribed to cDNA using the High Capacity cDNA Reverse Transcription Kit (Applied Biosystems, Thermo Fisher). PCR was used to amplify Tnnt3 amplicons representing all expressed splice forms as described previously [36]. Fluorescein (6FAM)-labeled Tnnt3 PCR products were diluted $1: 10$ and one $\mu \mathrm{L}$ per sample was analyzed by capillary electrophoresis as described previously [36]. Briefly, separated Tnnt3 amplicons were detected as individual fluorescence peaks of varying height within a range of $0-1200 \mathrm{bp}$. An internal size standard allowed accurate sizing of Tnnt 3 amplicons that were determined to vary between $\sim 700-800 \mathrm{bp}$. The relative abundance of each Tnnt 3 amplicon was then calculated by dividing the fluorescence peak height by the total of all Tnnt3 amplicon peak heights. The fold change in the relative abundance of each Tnnt3 splice form relative to controls was calculated for each experiment.

\section{Quantitative real-time PCR of Tnnt3 mRNA}

Quantitative real-time PCR (qRT-PCR) was performed using the QuantStudio $12 \mathrm{~K}$ Flex Real-Time PCR System and SYBR Green master mix (Qiagen, Germantown, MD) according to the manufacturer's instructions. Tnnt3 gene expression was quantified using rat specific primers designed to constitutively expressed (i.e., not alternatively spliced) Tnnt3 exons 11 and 12 (Fwd: 5' GTCA GAACAAGGACCTCATGG 3', Rev.: 5' TCTCAGCGC GAATCCTTTG 3') and a region within the gene Gapdh (Fwd: 5'-AGTTCAACGGCACAGTCAAG-3', Rev.: 5'-T ACTCAGCACCAGCATCACC-3'). Changes in Tnnt3 gene expression were normalized to Gapdh using the $2^{-\Delta \Delta C_{T}}$ method as described previously [38].

\section{Statistical analysis}

Tnnt3 splice form and Western blot analyses are displayed as the mean fold change \pm SEM of the relative abundance or ratio of phosphorylated/total protein, respectively, compared to controls. Fold change values were calculated from each of three independent experiments with three replicates per experiment. GraphPad Prism v7.0 was used to generate graphs and to perform Student's t-tests or One- or Two-way ANOVA with Fisher's LSD post-hoc test for multiple comparisons to determine statistical significance $(p \leq 0.05)$.

\section{Results}

\section{Characterization of L6 myotube Tnnt3 splice form expression}

Skeletal muscle contractile elements, including Tnnt3, are expressed in differentiated mouse $(\mathrm{C} 2 \mathrm{C} 12)$ myotubes [36, 39], but to our knowledge a comprehensive analysis of Tnnt3 splice forms had not been performed previously in rat L6 myotubes. Thus, we induced L6 myoblasts to differentiate into myotubes and analyzed Tnnt3 total mRNA, protein, and splice form expression at day 0 (D0), and day 8 (D8) post-differentiation onset. Myoblasts fused to form mature myotubes (Fig. 1a) and total Tnnt3 mRNA abundance, as assessed by qRT-PCR, was increased 76-fold by D8 (Fig. 1b). A similar effect was observed with TNNT3 protein expression (Fig. 1c). Capillary electrophoresis of Tnnt3 PCR amplicons detected three Tnnt3 splice forms present in low abundance at D0 (Fig. 1d). Expression of all three splice forms present in undifferentiated myoblasts increased after differentiation, and 15 splice forms not detected in myoblasts were present in D8 myotubes (Fig. 1e).

\section{In vitro modulation of Tnnt3 alternative splicing by fatty acids and ceramides}

We next tested if exposure to fatty acids could modulate alternative splicing of Tnnt3 in L6 myotubes. Cells were treated with BSA conjugated to saturated (palmitate), mono- (oleate) and poly-unsaturated (linoleate) fatty acids that were also present in the diets of our previous study [19], or with BSA alone for $24 \mathrm{~h}$. Treatment with oleate or linoleate significantly altered the expression of the 781 and $795 \mathrm{bp}$ Tnnt3 splice forms compared to BSA-treated myotubes, however the magnitude of the effect was small (i.e., 1.2-fold or less; Table 1). The splice form exhibiting the largest change was the $737 \mathrm{bp}$ Tnnt3 splice form in response to palmitate treatment. This effect was specific to palmitate as it caused a 1.8-fold increase in relative abundance compared to treatment with BSA alone while neither oleic nor linoleic acid treatment had any effect on its relative abundance (Fig. 2a and Table 1). The $737 \mathrm{bp}$ Tnnt3 splice form was the most consistently and robustly changed splice form across 
a

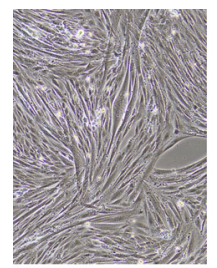

C

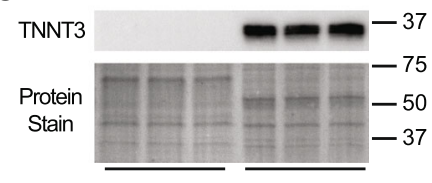

b

d

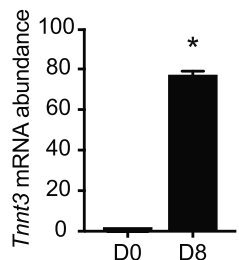

D8
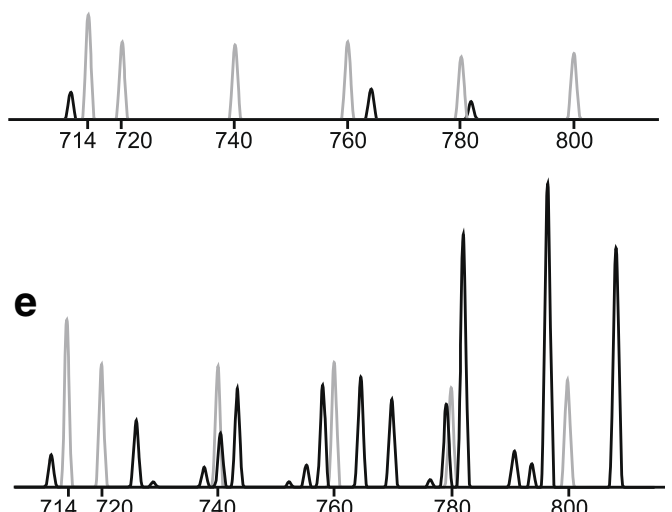

Fig. 1 Characterization of Tnnt3 mRNA, protein, and splice form expression in differentiating L6 myotubes. L6 myotubes were collected at the onset of differentiation (D0) or eight days post differentiation (D8) as described in Methods. (a) Micrograph of D8 L6 myotubes. (b) Tnnt3 mRNA abundance was assessed by qRT-PCR and normalized to Gapdh. (c) TNNT3 protein expression was assessed by Western blot analysis. Protein staining of the Western blot membrane is shown to demonstrate equal protein loading. Capillary electrophoresis tracings of Tnnt3 splice forms from (d) D0 or (e) D8 of differentiation as assessed by fragment analysis of Tnnt3 PCR amplicons. Black and gray tracings represent Tnnt3 splice forms and internal size standards, respectively. Peak height is proportional to the splice form relative abundance. Data in (b) are presented as means \pm SEM from three independent experiments using three replicates per time point. Statistically different means as assessed by Student's t-test are denoted with an asterisk $\left(^{*}\right)(p \leq 0.05)$

Table 1 Fold change in the relative abundance of Tnnt3 splice forms

\begin{tabular}{|c|c|c|c|c|}
\hline Tnnt3 splice form size (bp) & BSA & PA & $\mathrm{OA}$ & $\mathrm{LA}$ \\
\hline 710 & $1.00^{a}$ & $1.228 \pm 0.240^{a}$ & $1.042 \pm 0.099^{a}$ & $1.091 \pm 0.067^{\mathrm{a}}$ \\
\hline 725 & $1.00^{a, c}$ & $1.212 \pm 0.109^{a}$ & $0.970 \pm 0.041^{b, c}$ & $1.022 \pm 0.078^{a}$ \\
\hline 728 & $1.00^{\mathrm{a}}$ & $0.713 \pm 0.098^{a}$ & $0.832 \pm 0.239^{a}$ & $0.875 \pm 0.270^{a}$ \\
\hline 737 & $1.00^{\mathrm{a}}$ & $1.851 \pm 0.143^{b}$ & $0.943 \pm 0.053^{a}$ & $0.921 \pm 0.180^{a}$ \\
\hline 739 & $1.00^{\mathrm{a}}$ & $1.438 \pm 0.202^{a}$ & $1.327 \pm 0.350^{\mathrm{a}}$ & $1.115 \pm 0.403^{a}$ \\
\hline 742 & $1.00^{\mathrm{a}}$ & $1.026 \pm 0.057^{\mathrm{a}}$ & $0.946 \pm 0.044^{a}$ & $0.978 \pm 0.117^{a}$ \\
\hline 751 & $1.00^{\mathrm{a}}$ & $1.198 \pm 0.209^{a}$ & $0.662 \pm 0.247^{a}$ & $0.794 \pm 0.063^{a}$ \\
\hline 754 & $1.00^{a, b}$ & $1.233 \pm 0.177^{\mathrm{a}}$ & $0.764 \pm 0.150^{b}$ & $0.826 \pm 0.069^{b}$ \\
\hline 757 & $1.00^{a}$ & $1.105 \pm 0.061^{a}$ & $0.923 \pm 0.091^{\mathrm{a}}$ & $1.013 \pm 0.086^{a}$ \\
\hline 763 & $1.00^{\mathrm{a}}$ & $0.989 \pm 0.104^{a}$ & $0.860 \pm 0.097^{\mathrm{a}}$ & $0.884 \pm 0.069^{a}$ \\
\hline 769 & $1.00^{a}$ & $1.071 \pm 0.036^{a}$ & $0.906 \pm 0.124^{a}$ & $0.804 \pm 0.112^{a}$ \\
\hline 775 & $1.00^{\mathrm{a}}$ & $1.334 \pm 0.177^{a}$ & $0.990 \pm 0.233^{a}$ & $0.977 \pm 0.254^{\mathrm{a}}$ \\
\hline 778 & $1.00^{\mathrm{a}}$ & $0.999 \pm 0.164^{a}$ & $1.151 \pm 0.017^{a}$ & $1.085 \pm 0.119^{a}$ \\
\hline 781 & $1.00^{\mathrm{a}}$ & $0.950 \pm 0.030^{a}$ & $1.104 \pm 0.014^{b}$ & $1.114 \pm 0.033^{b}$ \\
\hline 790 & $1.00^{a}$ & $0.839 \pm 0.070^{a}$ & $0.833 \pm 0.042^{a}$ & $0.790 \pm 0.157^{a}$ \\
\hline 793 & $1.00^{\mathrm{a}}$ & $0.837 \pm 0.036^{b, c}$ & $0.935 \pm 0.054^{a, c}$ & $0.776 \pm 0.078^{b}$ \\
\hline 795 & $1.00^{a, b}$ & $0.953 \pm 0.044^{a}$ & $1.071 \pm 0.043^{b}$ & $1.216 \pm 0.078^{c}$ \\
\hline 807 & $1.00^{a}$ & $0.966 \pm 0.030^{a, b}$ & $0.955 \pm 0.035^{a, b}$ & $0.909 \pm 0.020^{b}$ \\
\hline
\end{tabular}

L6 myotubes were treated for $24 \mathrm{~h}$ with $150 \mu \mathrm{M}$ palmitate, oleate, or linoleate (PA, OA, LA, respectively) conjugated to BSA or an equal volume of BSA alone. The fold change in the relative abundance of Tnnt3 splice forms was assessed by capillary electrophoresis. Data are presented as means \pm SEM from three independent experiments using three replicates per treatment. Statistical significance was assessed by One-way ANOVA and Fishers LSD post-hoc test for multiple comparisons. Statistically different means are denoted with different letters $(p \leq 0.05)$ 

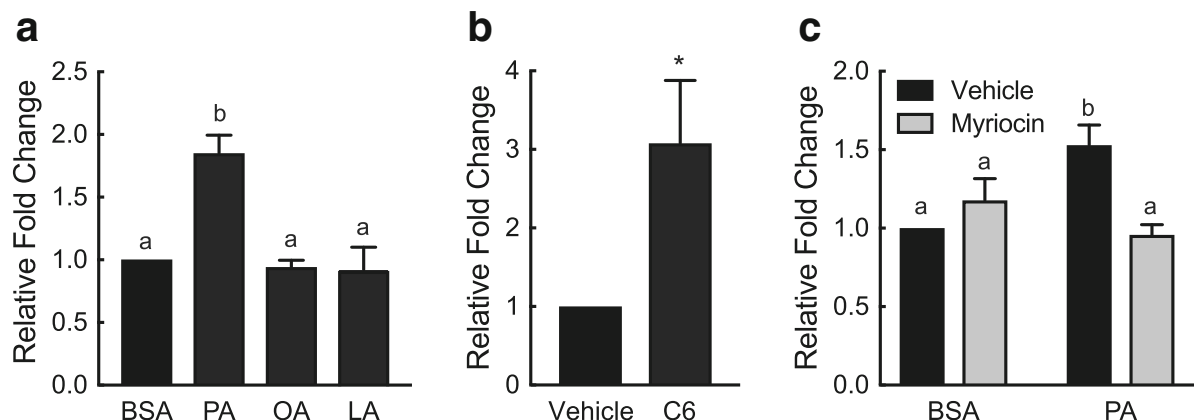

Fig. 2 Fatty acid- and C6 ceramide-induced changes in Tnnt3 pre-mRNA alternative splicing. L6 myotubes were treated for $24 \mathrm{~h}$ with (a) $150 \mu \mathrm{M}$ palmitate, oleate, or linoleate (PA, OA, LA, respectively) conjugated to BSA or an equal volume of BSA alone, (b) $20 \mu \mathrm{M}$ C6 ceramide (C6) or an equal volume of methanol (Vehicle), or (c) $50 \mathrm{nM}$ myriocin or an equal volume of methanol (Vehicle) for two hours prior to a 24-h treatment with 150 $\mathrm{\mu M}$ PA bound to BSA (PA) or an equal volume of BSA alone. The fold change in the relative abundance of the 737 base pair Tnnt3 splice form was assessed by capillary electrophoresis. Data are presented as means \pm SEM from three independent experiments using three replicates per treatment. Statistical significance was assessed by Student's t-test (b) and One- and Two-way ANOVA with Fishers LSD post-hoc test for multiple comparisons (a and $\mathbf{c}$, respectively). Statistically different means are denoted with an asterisk (*) or different letters above the bars $(p \leq 0.05)$

studies. Moreover, the most abundant splice form in adult rat loading bearing muscle, i.e. the gastrocnemius, is the $737 \mathrm{bp}$ splice form [34]. Therefore, for ease of presentation, from here onward data for all 18 Tnnt3 splice forms will be presented in table format as Additional Files and data for the $737 \mathrm{bp}$ Tnnt3 splice form will be presented in graphical format.

Palmitate is a substrate for serine palmitoyltransferase, the initial and rate limiting step in de novo ceramide biosynthesis [40]. Thus, the palmitate-induced effects on Tnnt3 alternative splicing may be due to de novo biosynthesis of ceramides. Indeed, a 24-h treatment with 20 $\mu \mathrm{M}$ C6 ceramide increased the relative abundance of the $737 \mathrm{bp}$ Tnnt3 splice form by 2.7 -fold (Fig. $2 \mathrm{~b}$ and Additional file 1). Additionally, C6 ceramide treatment significantly altered the relative abundance of 6 of the 18 Tnnt3 splice forms (Additional file 1). Pre-treatment with myriocin, a selective serine palmitoyltransferase inhibitor which blocks the initial, crucial catalytic step of ceramide biosynthesis, attenuated the palmitate-induced increase in the relative abundance of the $737 \mathrm{bp}$ Tnnt 3 splice form (Fig. 2c and Additional file 2). These data demonstrate that both fatty acids and ceramides induce changes in Tnnt 3 alternative splicing and that the effect of palmitate likely requires de novo ceramide synthesis.

In vitro modulation of Akt S473 phosphorylation by fatty acids and ceramides

We next sought to gain insight into possible signaling pathways involved in the palmitate- and ceramide-induced
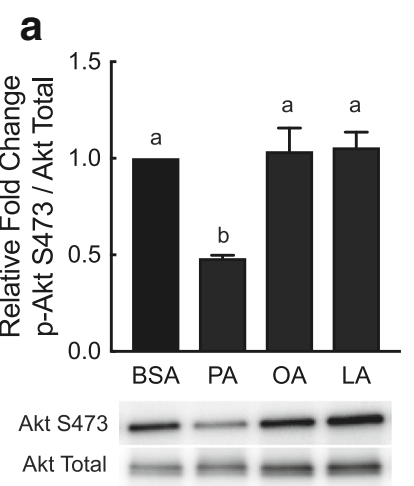

\section{b}

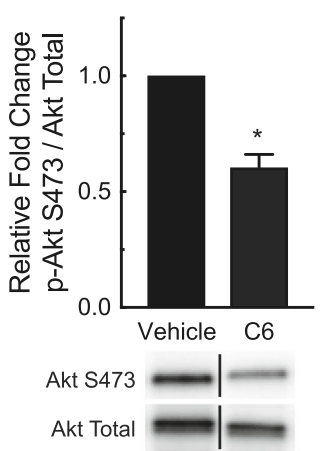

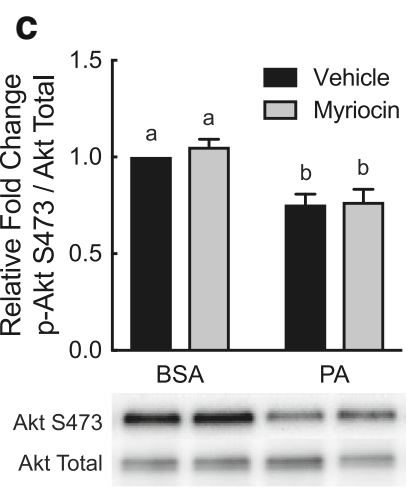

Fig. 3 Fatty acid- and C6 ceramide-induced changes in Akt S473 phosphorylation. L6 myotubes were treated for $24 \mathrm{~h}$ with (a) $150 \mu \mathrm{M}$ palmitate, oleate, or linoleate (PA, OA, LA, respectively) conjugated to BSA or an equal volume of BSA alone, (b) $20 \mu \mathrm{M}$ C6 ceramide (C6) or an equal volume of methanol (Vehicle), or (c) $50 \mathrm{nM}$ myriocin or an equal volume of methanol (Vehicle) for two hours prior to a 24-h treatment with $150 \mu \mathrm{M}$ PA bound to BSA or an equal volume of BSA alone. The ratio of phosphorylated Akt at S473 to total Akt was assessed by Western blot analysis. Black lines in the Western blot images shown in B represent noncontiguous lanes from the same blot. Data are presented as means \pm SEM from three independent experiments using three replicates per treatment. Statistical significance was assessed by Student's t-test (b) and One- and Two-way ANOVA with Fishers LSD post-hoc test for multiple comparisons (a and $\mathbf{c}$, respectively). Statistically different means are denoted with an asterisk $\left(^{*}\right)$ or different letters above the bars $(p \leq 0.05)$ 
effects on Tnnt 3 alternative splicing. Therefore, we assessed whether Akt signaling correlated with changes to the relative abundance of the $737 \mathrm{bp}$ Tnnt3 splice form because a hallmark effect of ceramides is their ability to alter Akt phosphorylation [23, 41] and our previous findings in $\mathrm{C} 2 \mathrm{C} 12$ myotubes suggested an important role for Akt in modulating Tnnt3 pre-mRNA alternative splicing [36]. As shown in Fig. 3a, palmitate, but not oleate or linoleate, reduced Akt S473 phosphorylation compared to BSA-treated L6 myotubes (Fig. 3a). Moreover, treatment with C6 ceramide reduced Akt $\mathrm{S} 473$ phosphorylation by $39.5 \pm$ $5.6 \%$, compared to controls (Fig. 3b). Interestingly, although palmitate treatment significantly reduced Akt S473 phosphorylation, pretreatment with myriocin did not prevent the palmitate-induced reduction (Fig. 3c), suggesting that palmitate is either not acting through Akt or acts downstream of Akt to modulate alternative splicing.
Inhibition of PP2A attenuates the palmitate-and ceramide-induced alternative splicing of Tnnt3

A major mode of action of ceramides is the activation of PP1 and PP2A [16, 41]. Because changes in Akt S473 phosphorylation, a PP1 specific site [42], did not explain the palmitate-induced effects on Tnnt3 alternative splicing, we hypothesized that palmitate and C6 ceramide exert their effects on Tnnt3 alternative splicing in part through the activation of PP2A. A previous study [43] showed that $15 \mathrm{nM}$ okadaic acid selectively inhibited PP2A in primary cultures of rat hepatocytes. Confirming that finding, we found that in L6 myotubes, $15 \mathrm{nM}$ okadaic acid significantly increased the phosphorylation of the PP2A substrates GSK-3 $\beta$ [44] and ERK1/2 [45] but not the PP1 substrate Akt S473 (Fig. 4a-c). Notably, pretreatment with $15 \mathrm{nM}$ okadaic acid blocked the effect of palmitate and C6 ceramide on the abundance of the $737 \mathrm{bp}$ Tnnt 3 splice form (Fig. 4d and e) and prevented or attenuated the change in abundance of several other
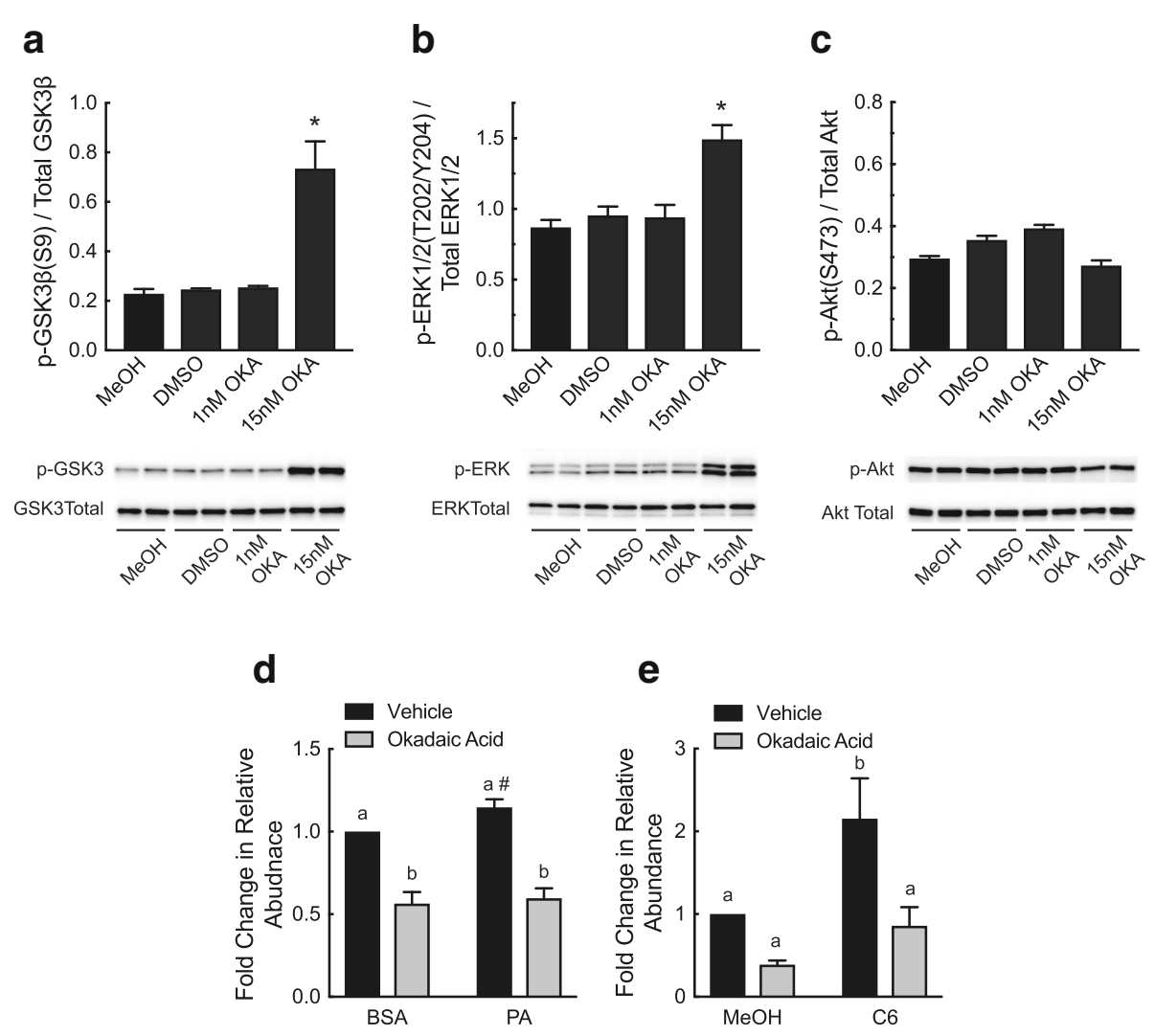

Fig. 4 Palmitate- and C6 ceramide-induced changes in Tnnt3 pre-mRNA alternative splicing are blocked by inhibition of PP2A. a-c L6 myotubes were pretreated for two hours with either 1 or $15 \mathrm{nM}$ okadaic acid (OKA) or an equal volume of MeOH or DMSO for 24-h prior to Western blot analysis. The ratio of (a) GSK3 $\beta$ at S9 to total GSK3 3 , (b) ERK1/2 at T202/Y204 to total ERK1/2, and (c) phosphorylated Akt at S473 to total Akt was assessed. L6 myotubes were pretreated for two hours with okadaic acid or an equal volume of DMSO (Vehicle) prior to a 24-h treatment with (d) $150 \mu \mathrm{M}$ PA conjugated to BSA (PA) or an equal volume of BSA alone or (e) $20 \mu \mathrm{M}$ C6 ceramide (C6) or an equal volume of methanol (MeOH). The fold change in the relative abundance of the 737 base pair Tnnt3 splice form was assessed by capillary electrophoresis (d and $\mathbf{e}$ ). Data are presented as means \pm SEM from three independent experiments using three replicates per treatment. Statistical significance was assessed by Student's t-test (a-c) or Two-way ANOVA with Fishers LSD post-hoc test for multiple comparisons ( $\mathbf{d}$ and $\mathbf{e})$. Statistically different means are denoted with an asterisk $\left(^{*}\right)$ or different letters above the bars $(p \leq 0.05)$. \# $p=0.08$ vs. BSANehicle 
Tnnt3 splice forms (Additional files 3 and 4). Thus, these data suggest that the effects of palmitate and C6 ceramide on the alternative splicing of the $737 \mathrm{bp}$ Tnnt3 splice form expression occurs in a PP2A-dependent manner.

\section{Discussion}

The results of the present study support a model in which saturated fatty acids increase ceramide expression leading to the activation of PP2A and subsequent changes in the alternative splicing of Tnnt3 pre-mRNA in L6 myotubes. In support of this model, the data show that both palmitate and $\mathrm{C} 6$ ceramide promote accumulation of the $737 \mathrm{bp}$ Tnnt 3 splice form and that the effect of palmitate is blocked by the ceramide synthesis inhibitor, myriocin. Although we did not measure ceramide levels in this study, others have demonstrated a timeand dose-dependent increase in ceramide accumulation in palmitate-treated cells in culture [46] and that the increase is blocked by myriocin treatment [20]. Thus, the repressive effect of myriocin on palmitate-induced Tnnt3 alternative splicing is consistent with the fatty acid acting through a mechanism requiring increased ceramide synthesis to produce changes in alternative splicing. Importantly, previous studies have demonstrated that increased ceramide production is associated with changes in alternative splicing of pre-mRNAs other than Tnnt3. For example, both TNF $\alpha$ and gemcitabine have been reported to alter the pattern of pre-mRNA alternative splicing of PKC $B \mathrm{II}$, caspase 9 , and $\mathrm{Bcl}-\mathrm{xL}$ and myriocin blocks the effect $[23,24]$, suggesting that, like palmitate, TNF $\alpha$ and gemcitabine-induced changes in alternative splicing require de novo ceramide synthesis.

In the present study, we also found that fatty acids of varying saturation level elicit distinct patterns of Tnnt3 pre-mRNA alternative splicing. Notably, in several instances, the pattern of Tnnt3 alternative splicing was different in myotubes treated with the unsaturated fatty acids oleate and linoleate, compared to those treated with palmitate. For example, treatment with either oleate or linoleate elicited differential effects on the expression of the 793 and $795 \mathrm{bp}$ Tnnt 3 splice forms (Table 1). The effect of oleate or linoleate is most likely independent of increased ceramide production because, unlike palmitate, they have no effect on ceramide production or accumulation in skeletal muscle [47] or in cells in culture [48-50]. Instead, it is possible that there are ceramide-independent mechanisms that lead to variations in the Tnnt 3 splice form expression in response to treatment with unsaturated fatty acids. However, the magnitude of the changes in alternative splicing induced by unsaturated fatty acids was small, and the physiological significance of such relatively small changes must be questioned.
An important mechanism for regulating alternative splicing involves changes in phosphorylation of the SR proteins that belong to the non-small nuclear ribonucleoprotein particle splicing factor family of proteins [4, 11-14]. The SR proteins are phosphorylated on multiple serine residues by various kinases including Akt, leading to changes in the alternative splicing process [12, 14]. In the present study, Akt phosphorylation on S473 was reduced in response to treatment with either palmitate or C6 ceramide in association with increased Tnnt3 737 abundance, consistent with a possible role for altered Akt signaling in mediating the observed changes in alternative splicing. However, although myriocin blocked the palmitate-induced changes in alternative splicing, it did not prevent the changes in Akt S473 phosphorylation, suggesting that the changes in alternative splicing occurred in an Akt-independent manner. Instead, okadaic acid when used at a concentration that inhibits PP2A but not PP1 blocked the effect of both palmitate and ceramide on alternative splicing of the Tnnt3 737 splice form. Previous studies have shown that ceramide treatment leads to activation of both PP1 and PP2A [51-53]. Thus, it is tempting to speculate that palmitate-induced ceramide production leads to activation of PP2A and subsequent modulation of Tnnt3 alternative splicing.

It is interesting that treatment with C6 ceramide had a more dramatic effect on Tnnt3 alternative splicing compared to palmitate, both with regard to the number of splice forms affected and, in some cases, the magnitude of the change. Although the basis for this difference is unknown, it is noteworthy that C6 ceramide is cell permeable and does not need to be bound to BSA to be soluble in aqueous solution [24]. In contrast, palmitate was bound to BSA and requires fatty acid transport proteins to enter the cell [54]. Thus, C6 ceramide likely enters the cell more rapidly compared to palmitate, and may reach higher intracellular concentrations more quickly compared to the ceramide generated by palmitate-induced increases in synthesis. It is also possible that the shorter chain ceramides like C6 ceramide have a differential effect to promote alternative splicing compared to longer chain ones.

\section{Conclusion}

In addition to acting as energy sources and structural components of cells, fatty acids and their metabolites act as signaling molecules to regulate gene expression. The data presented in the current study extend the biological significance of fatty acids by demonstrating that fatty acids and their metabolites play a role in the selection of exons for alternative splicing and uncover an important node in the nutrient control of gene expression. The 
basic mechanisms of pre-mRNA alternative splicing have been studied extensively $[10,55]$. However, surprisingly, how alternative splicing regulatory mechanisms are influenced by changes in environmental conditions or nutrient availability has only recently been investigated $[19,56,57]$. Approximately $95 \%$ of all multi-exon pre-mRNAs are thought to generate multiple mature mRNA splice forms [3], therefore future studies should expand upon the list of pre-mRNAs affected by nutrient availability, the signaling pathways involved, and the functional outcomes of these changes.

\section{Additional files}

Additional file 1: Fold change in the relative abundance of Tnnt3 splice forms in L6 myotubes treated with C6 ceramide. (DOCX $15 \mathrm{~kb}$ )

Additional file 2: Fold change in the relative abundance of Tnnt3 splice forms in L6 myotubes treated with myriocin. (DOCX $16 \mathrm{~kb}$ )

Additional file 3: Fold change in the relative abundance of Tnnt3 splice forms in $\mathrm{L} 6$ myotubes treated with okadaic acid and palmitate. (DOCX $16 \mathrm{~kb})$

Additional file 4: Fold change in the relative abundance of Tnnt3 splice forms in L6 myotubes treated with okadaic acid and C6 ceramide. (DOCX 16 kb)

\section{Abbreviations}

BCl-xL: B-cell lymphoma extra-long; bp: base pairs; BSA: bovine serum albumin; DM: differentiation medium; ERK: extracellular signal-regulated kin-

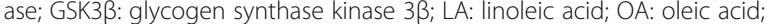
PA: palmitic acid; PP: protein phosphatase; SR: serine-arginine-rich; TNFa: tumor necrosis factor a; Tnnt3: troponin T3

\section{Acknowledgements}

We gratefully acknowledge Holly Lacko and Lydia Kutzler for their technical assistance in performing portions of the studies described in this manuscript and Dr. Leonard S. Jefferson for critical reading of the manuscript.

\section{Funding}

This research was supported by NIH grant R01-DK-09141 (to SRK) and NSF grant IOS 1457237 (to RJS).

\section{Availability of data and materials}

All data generated or analyzed during this study are included in this published article [and its supplementary information files].

\section{Authors' contributions}

AJB and SRK designed experiments, AJB performed experiments and wrote the manuscript, RJS and SRK participated in manuscript preparation. All authors have approved the final version of the manuscript.

\section{Ethics approval and consent to participate}

Not applicable (no humans or animals).

\section{Consent for publication}

All authors consent to publication of the data.

\section{Competing interests}

The authors declare they have no competing interests.

\section{Publisher's Note}

Springer Nature remains neutral with regard to jurisdictional claims in published maps and institutional affiliations.

\section{Author details}

'Department of Cellular and Molecular Physiology, Penn State College of Medicine, H166, 500 University Drive, Hershey, PA 17033, USA. ${ }^{2}$ Present Address: Department of Cell Biology and Physiology, 6330 Medical Biomolecular Research Building, 111 Mason Farm Rd, Chapel Hill, NC 27599, USA. ${ }^{3}$ Department of Entomology and Biology, Penn State University, University Park, PA, USA.

Received: 22 August 2018 Accepted: 10 December 2018

Published online: 17 December 2018

\section{References}

1. Baralle FE, Giudice J. Alternative splicing as a regulator of development and tissue identity. Nat Rev Mol Cell Biol. 2017;18:437-51.

2. Bortfeldt R, Schindler S, Szafranski K, Schuster S, Holste D. Comparative analysis of sequence features involved in the recognition of tandem splice sites. BMC Genomics. 2008;9:202.

3. Lee $Y$, Rio DC. Mechanisms and regulation of alternative pre-mRNA splicing. Annu Rev Biochem. 2015;84:291-323.

4. Naro C, Sette C. Phosphorylation-mediated regulation of alternative splicing in cancer. Int J Cell Biol. 2013;2013:151839.

5. Castle JC, Zhang C, Shah JK, Kulkarni AV, Kalsotra A, Cooper TA, Johnson JM. Expression of 24,426 human alternative splicing events and predicted cis regulation in 48 tissues and cell lines. Nat Genet. 2008:40:1416-25.

6. Matera AG, Wang Z. A day in the life of the spliceosome. Nat Rev Mol Cell Biol. 2014;15:108-21.

7. Szeszel-Fedorowicz W, Talukdar I, Griffith BN, Walsh CM, Salati LM. An exonic splicing silencer is involved in the regulated splicing of glucose 6phosphate dehydrogenase mRNA. J Biol Chem. 2006;281:34146-58.

8. Bland CS, Wang ET, Vu A, David MP, Castle JC, Johnson JM, Burge CB, Cooper TA. Global regulation of alternative splicing during myogenic differentiation. Nucleic Acids Res. 2010;38:7651-64.

9. Huelga SC, Vu AQ, Arnold JD, Liang TY, Liu PP, Yan BY, Donohue JP, Shiue L, Hoon S, Brenner $S$, et al. Integrative genome-wide analysis reveals cooperative regulation of alternative splicing by hnRNP proteins. Cell Rep. 2012;1:167-78.

10. Sebbag-Sznajder N, Raitskin O, Angenitzki M, Sato TA, Sperling J, Sperling R. Regulation of alternative splicing within the supraspliceosome. J Struct Biol. 2012;177:152-9.

11. Sanidas I, Polytarchou C, Hatziapostolou M, Ezell SA, Kottakis F, Hu L, Guo A, Xie J Comb M, Iliopoulos D, Tsichlis PN. Phosphoproteomics screen reveals akt isoformspecific signals linking RNA processing to lung cancer. Mol Cell. 2014;53:577-90.

12. Zhou Z, Qiu J, Liu W, Zhou Y, Plocinik RM, Li H, Hu Q, Ghosh G, Adams JA, Rosenfeld MG, Fu XD. The Akt-SRPK-SR axis constitutes a major pathway in transducing EGF signaling to regulate alternative splicing in the nucleus. Mol Cell. 2012:47:422-33

13. Zhu C, Yin Z, Tan B, Guo W. Insulin regulates titin pre-mRNA splicing through the PI3K-Akt-mTOR kinase axis in a RBM20-dependent manner. Biochim Biophys Acta. 2017:1863:2363-71.

14. Blaustein M, Pelisch F, Tanos T, Munoz MJ, Wengier D, Quadrana L, Sanford $J R$, Muschietti JP, Kornblihtt AR, Caceres JF, et al. Concerted regulation of nuclear and cytoplasmic activities of SR proteins by AKT. Nat Struct Mol Biol. 2005;12:1037-44.

15. Shi Y, Reddy B, Manley JL. PP1/PP2A phosphatases are required for the second step of pre-mRNA splicing and target specific snRNP proteins. Mol Cell. 2006:23:819-29.

16. Chalfant CE, Ogretmen B, Galadari S, Kroesen BJ, Pettus BJ, Hannun YA. FAS activation induces dephosphorylation of SR proteins; dependence on the de novo generation of ceramide and activation of protein phosphatase 1. J Biol Chem. 2001:276:44848-55.

17. Michlewski G, Sanford JR, Caceres JF. The splicing factor SF2/ASF regulates translation initiation by enhancing phosphorylation of 4E-BP1. Mol Cell. 2008;30:179-89.

18. Johnston JR, Chase PB, Pinto JR. Troponin through the looking-glass: emerging roles beyond regulation of striated muscle contraction. Oncotarget. 2018;9:1461-82.

19. Black AJ, Ravi S, Jefferson LS, Kimball SR, Schilder RJ. Dietary fat quantity and type induce transcriptome-wide effects on alternative splicing of pre-mRNA in rat skeletal muscle. J Nutr. 2017:147:1648-57.

20. Miklosz A, Lukaszuk B, Baranowski M, Gorski J, Chabowski A. Effects of inhibition of serine palmitoyltransferase (SPT) and sphingosine kinase 1 (SphK1) on palmitate induced insulin resistance in L6 myotubes. PLoS One. 2013;8:e85547. 
21. Blachnio-Zabielska AU, Chacinska M, Vendelbo MH, Zabielski P. The crucial role of C18-Cer in fat-induced skeletal muscle insulin resistance. Cell Physiol Biochem. 2016;40:1207-20.

22. Nikolova-Karakashian MN, Reid MB. Sphingolipid metabolism, oxidant signaling, and contractile function of skeletal muscle. Antioxid Redox Signal. 2011;15:2501-17

23. Ghosh N, Patel N, Jiang K, Watson JE, Cheng J, Chalfant CE, Cooper DR. Ceramide-activated protein phosphatase involvement in insulin resistance via Akt, serine/arginine-rich protein 40, and ribonucleic acid splicing in L6 skeletal muscle cells. Endocrinology. 2007;148:1359-66.

24. Chalfant CE, Rathman K, Pinkerman RL, Wood RE, Obeid LM, Ogretmen B, Hannun YA. De novo ceramide regulates the alternative splicing of caspase 9 and $\mathrm{BCl}-\mathrm{x}$ in A549 lung adenocarcinoma cells. Dependence on protein phosphatase-1. J Biol Chem. 2002;277:12587-95.

25. Brandimarti P, Costa-Junior JM, Ferreira SM, Protzek AO, Santos GJ, Carneiro EM, Boschero AC, Rezende LF. Cafeteria diet inhibits insulin clearance by reduced insulin-degrading enzyme expression and mRNA splicing. J Endocrinol. 2013;219:173-82.

26. Kim Y, Tamura T, Iwashita S, Tokuyana K, Suzuki M. Effect of high-fat diet on gene expression of GLUT4 and insulin receptor in soleus muslce. Biochem Biophys Res Commun. 1994;202:519-26.

27. Marden JH, Fescemyer HW, Saastamoinen M, MacFarland SP, Vera JC, Frilander MJ, Hanski I. Weight and nutrition affect pre-mRNA splicing of a muscle gene associated with performance, energetics and life history. J Exp Biol. 2008;211:3653-60

28. Tao H, Szeszel-Fedorowicz W, Amir-Ahmady B, Gibson MA, Stabile LP, Salati LM. Inhibition of the splicing of glucose-6-phosphate dehydrogenase precursor mRNA by polyunsaturated fatty acids. J Biol Chem. 2002;277: 31270-8.

29. Walsh CM, Suchanek AL, Cyphert TJ, Kohan AB, Szeszel-Fedorowicz W, Salati $L M$. Serine arginine splicing factor 3 is involved in enhanced splicing of glucose-6-phosphate dehydrogenase RNA in response to nutrients and hormones in liver. J Biol Chem. 2013;288:2816-28.

30. Griffith BN, Walsh CM, Szeszel-Fedorowicz W, Timperman AT, Salati LM. Identification of hnRNPs $K, L$ and $A 2 / B 1$ as candidate proteins involved in the nutritional regulation of mRNA splicing. Biochim Biophys Acta. 2006; 1759:552-61.

31. Kaminska $D$, Hämäläinen $M$, Cederberg $H$, Käkelä $P$, Venesmaa $S$, Miettinen P, Ilves I, Herzig KH, Kolehmainen M, Karhunen L, et al. Adipose tissue INSR splicing in humans associates with fasting insulin level and is regulated by weight loss. Diabetologia. 2014;57:347-51.

32. Kaminska D, Kuulasmaa T, Venesmaa $S$, Käkelä $P$, Vaittinen M, Pulkkinen L, Pääkkönen M, Gylling H, Laakso M, Pihlajamäki J. Adipose tissue TCF7L2 splicing is regulated by weight loss and associates with glucose and fatty acid metabolism. Diabetes. 2012;61:2807-13.

33. Kaminska D, Pihlajamaki J. Regulation of alternative splicing in obesity and weight loss. Adipocyte. 2013;2:143-7.

34. Schilder RJ, Kimball SR, Marden JH, Jefferson LS. Body weight-dependent troponin $T$ alternative splicing is evolutionarily conserved from insects to mammals and is partially impaired in skeletal muscle of obese rats. J Exp Biol. 2011;214:1523-32

35. Webster NJG, Huang Z. Hormonal regulation of alternative splicing. In: Chew SL, editor. Post-transcriptional processing and the endocrine system. New York: Karger; 1999. p. 1-17.

36. Schilder RJ, Kimball SR, Jefferson LS. Cell-autonomous regulation of fast troponin T pre-mRNA alternative splicing in response to mechanical stretch. Am J Physiol Cell Physiol. 2012;303:C298-307.

37. Black AJ, Gordon BS, Dennis MD, Jefferson LS, Kimball SR. Regulation of protein and mRNA expression of the mTORC1 repressor REDD1 in response to leucine and serum. Biochem Biophys Rep. 2016;8:296-301.

38. Livak KJ, Schmittgen TD. Analysis of relative gene expression data using real-time quantitative $P C R$ and the 2(-Delta Delta $C(T)$ ) method. Methods. 2001;25:402-8

39. Cabane C, Englaro W, Yeow K, Ragno M, Derijard B. Regulation of C2C12 myogenic terminal differentiation by MKK3/p38a pathway. Am J Physiol Cell Physiol. 2003;284:C658-C66.

40. Perry DK, Carton J, Shah AK, Meredith F, Uhlinger DJ, Hannun YA. Serine Palmitoyltransferase regulates de novo ceramide generation during etoposide-induced apoptosis. J Biol Chem. 2000;275:9078-84.

41. Mahfouz R, Khoury R, Blachnio-Zabielska A, Turban S, Loiseau N, Lipina C, Stretton C, Bourron O, Ferre P, Foufelle F, et al. Characterising the inhibitory actions of ceramide upon insulin signaling in different skeletal muscle cell models: a mechanistic insight. PLoS One. 2014;9:e101865.

42. Xu W, Yuan X, Jung YJ, Yang Y, Basso A, Rosen N, Chung EJ, Trepel J, Neckers L. The heat shock protein 90 inhibitor Geldanamycin and the ErbB inhibitor ZD1839 promote rapid PP1 phosphatase-dependent inactivation of AKT in ErbB2 overexpressing breast Cancer cells. Cancer Res. 2003;63:7777-84.

43. Holen I, Gordon PB, Seglen PO. Inhibition of hepatocytic autophagy by okadaic acid and other protein phosphatase inhibitors. Eur J Biochem. 1993; 215:113-22.

44. Hernandez F, Langa E, Cuadros R, Avila J, Villanueva N. Regulation of GSK3 isoforms by phosphatases PP1 and PP2A. Mol Cell Biochem. 2010;344:211-5.

45. Liu Q, Hofmann PA. Protein phosphatase 2A-mediated cross-talk between p38 MAPK and ERK in apoptosis of cardiac myocytes. Am J Physiol Heart Circ Physiol. 2004;286:H2204-H12.

46. Watt MJ, Barnett AC, Bruce CR, Schenk S, Horowitz JF, Hoy AJ. Regulation of plasma ceramide levels with fatty acid oversupply: evidence that the liver detects and secretes de novo synthesised ceramide. Diabetologia. 2012;55:2741-6.

47. Thombare K, Ntika S, Wang X, Krizhanovskii C. Long chain saturated and unsaturated fatty acids exert opposing effects on viability and function of GLP-1-producing cells: mechanisms of lipotoxicity. PLoS One. 2017;12: e0177605.

48. Holland WL, Bikman BT, Wang LP, Yuguang G, Sargent KM, Bulchand S, Knotts TA, Shui G, Clegg DJ, Wenk MR, et al. Lipid-induced insulin resistance mediated by the proinflammatory receptor TLR4 requires saturated fatty acid-induced ceramide biosynthesis in mice. J Clin Invest. 2011;121:1858-70.

49. Holland WL, Brozinick JT, Wang LP, Hawkins ED, Sargent KM, Liu Y, Narra K, Hoehn KL, Knotts TA, Siesky A, et al. Inhibition of ceramide synthesis ameliorates glucocorticoid-, saturated-fat-, and obesity-induced insulin resistance. Cell Metab. 2007:5:167-79.

50. Zhang Y, Rao E, Zeng J, Hao J, Sun Y, Liu S, Sauter ER, Bernlohr DA, Cleary MP, Suttles J, Li B. Adipose fatty acid binding protein promotes saturated fatty acid-induced macrophage cell death through enhancing ceramide production. J Immunol. 2017;198:798-807.

51. Dobrowsky RT, Kamibayashi C, Mumby MC, Hannun YA. Ceramide activates heterotrimeric protein phosphatase 2A. J Biol Chem. 1993;268:15523-30.

52. Chalfant CE, Kishihawa K, Mumby MC, Kamibayashi C, Bielawska A, Hannun YA. Long chain ceramides activate protein phosphatase-1 and protein phosphatase-2A. J Biol Chem. 1999;274:20313-7.

53. Chalfant CE, Szulc Z, Roddy P, Bielawska A, Hannun YA. The structural requirements for ceramide activation of serine-threonine protein phosphatases. J Lipid Res. 2004:45:496-506.

54. Glatz JF, Luiken JJ. Fatty acids in cell signaling: historical perspective and future outlook. Prostaglandins Leukot Essent Fatty Acids. 2015;92:57-62.

55. Will CL, Luhrmann R. Splicing of a rare class of introns by the U12dependent spliceosome. Biol Chem. 2005;386:713-24.

56. Guan Y, Liang G, Martin GB, Guan LL. Functional changes in mRNA expression and alternative pre-mRNA splicing associated with the effects of nutrition on apoptosis and spermatogenesis in the adult testis. BMC Genomics. 2017;18:64.

57. Ravi S, Schilder RJ, Berg AS, Kimball SR. Effects of age and hindlimb immobilization and remobilization on fast troponin T precursor mRNA alternative splicing in rat gastrocnemius muscle. Appl Physiol Nutr Metab. 2016:41:142-9.

Ready to submit your research? Choose BMC and benefit from:

- fast, convenient online submission

- thorough peer review by experienced researchers in your field

- rapid publication on acceptance

- support for research data, including large and complex data types

- gold Open Access which fosters wider collaboration and increased citations

- maximum visibility for your research: over $100 \mathrm{M}$ website views per year

At $\mathrm{BMC}$, research is always in progress.

Learn more biomedcentral.com/submission 